\title{
Servicios digitales para bibliotecas universitarias: el caso de los Sistemas de Información Geográfica (SIG)
}

Digital services for academic libraries: the case of Geographic Information Systems

\section{Sara Martínez Cardama, Mercedes Caridad Sebastián}

Departamento de Biblioteconomía y Documentación. Universidad Carlos III de Madrid. Instituto Agustín Millares, Universidad Carlos III de Madrid. Calle Madrid, 126, 28903 Getafe, Madrid, \{smarti1|mercedes\}@bib.uc3m.es

\begin{abstract}
Resumen
Se analiza la presencia e impacto de los Sistemas de Información geográfica (SIG) en el área de Biblioteconomía y Documentación, particularmente en bibliotecas universitarias, a través de dos estudios de caso: el primero, destinado a identificar los requisitos y competencias en materia de información geográfica detectadas a través de ofertas laborales de Información y Documentación, y, el segundo, basado en la evaluación de servicios SIG existentes en bibliotecas universitarias. La metodología de ambos estudios se ha sustentado en el análisis de contenido de las páginas web. Por un lado, se estudiaron 109 ofertas relacionadas con Servicios de Información Geográfica, publicadas en http://jobs.code4lib.org/ entre 2007 y 2014. Se determinaron las titulaciones, perfiles, experiencia y competencias principales demandadas. En paralelo, se analizaron las bibliotecas de las 50 primeras universidades del Ranking de Shanghai en su versión 2013. Los criterios de evaluación fueron la existencia de un servicio de información geográfica específico en la biblioteca, el grado de implicación de la misma en su gestión, así como el tipo de asistencia o material ofrecido. Ambos estudios ofrecen resultados en relación, por un lado, a los empleadores y las competencias que demandan, y por otro, a los servicios SIG en bibliotecas y sus características. Los resultados ayudan a determinar la importancia de los servicios de información geográfica en las perspectivas profesionales del área de Biblioteconomía y Documentación, así como el papel del bibliotecario en su actual desarrollo.
\end{abstract}

Palabras clave: Bibliotecas universitarias. Servicios de Información Geográfica. SIG.

\section{Introducción}

La información geográfica y cartográfica siempre ha sido de interés para las bibliotecas. La tecnología ha transformado la prestación de todos los servicios bibliotecarios, entre ellos los denominados Servicios SIG (Servicios de Información Geográfica). Estos ofrecen interesantes perspectivas de hibridación, debido a los nuevos enfoques en las dinámicas de investigación (foco en los datos, por ejemplo) y suponen un

\begin{abstract}
The presence and impact of Geographic Information Systems (GIS) in Library and Information Science is analyzed, particularly in academic libraries. The results of the two related case studies are presented. The first study was aimed at identifying GIS skills and requirements by examining LIS job offers. The second consisted of an evaluation of existent GIS services in academic libraries. In both cases the methodology was based on a content study through websites. On the one hand, 109 GIS job offers between 2007 and 2014 published in http://jobs.code4lib.org were analyzed, in particular the type of required qualifications, professional profiles, experience and main skills. On the other hand, the academic libraries of the top 50 universities of the ARWU (Academic Ranking of World Universities, 2013), researching the existence of a GIS service related to the academic library, the degree of implication of the library in it and the kind of assistance and material offered. Both studies gave intermingled results, in relation to the employer's demands and the characteristics of the GIS services in academic libraries. The results help to emphasize the importance of GIS for the Library and Information Science profession, as well as the librarian role in their current development.
\end{abstract}

Keywords: Academic libraries. Geographic Information System Services. GIS.

reto para la formación y estructuración de nuevos servicios bibliotecarios.

En materia de Servicios de Información Geográfica, no se habla ya de procesos de digitalización de documentos cartográficos, sino de elementos digitales ab initio. Son documentos dinámicos, que representan al extremo la importancia de las coordenadas tiempo-espacio en el manejo de la información, pues llevan asociados posiciones y son capaces de conectarse con 
otros datos que puestos sobre un mapa base aportan mayor información (Granell y Aguilar, 2013). En definitiva, es información con una gran capacidad de interacción que puede reutilizarse y crearse a medida del usuario.

Las definiciones de los Sistemas de Información Geográfica no solo ponen de relieve su carácter tecnológico, sino que se centran en la dimensión global de servicio. Así, en 2006 Aufmuth definió SIG, como

la infraestructura (hardware, software, personal...) los datos espaciales y las aplicaciones empleadas para inventarlas, gestionar y analizar datos geográficos.

Los factores en liza que supone la implantación de estos servicios para las bibliotecas ya los plantearon Weimer y Reehling en 2006, quienes señalaban que las bibliotecas no podrían de manera solitaria gestionar colecciones cartográficas de este nivel manteniéndolos en repositorios junto con otro tipo de colecciones. Plantearon, por tanto, una serie de requisitos curriculares más extensos que la gestión de colecciones digitales. Esta especificidad se muestra a continuación a través de las demandas que todo Servicio de Información Geográfica debería de cubrir (Houser, 2006; apud Kelly, 2013):

- Capacidad para descubrir datasets específicos. Houser plantea una hipotética consulta de información del tipo ("Límites administrativos de la Unión Soviética" o "mapas específicos sobre los destrozos del Katrina en Nueva Orleans").

- Generar mapas o imágenes a partir de datos geoespaciales.

- Convertir datos a pesar de la variedad de formatos específicos (vectorial, raster).

- Establecer subcategorías de datos, así como permitir añadir capas de los mismos.

- Permitir crear datos (establecer en el SW nuevos puntos, líneas o características) y posteriormente permitir editar la información (atributos) asociada.

- Mapear datos usando coordenadas geográficas para puntos concretos mediante su agregación a una capa SIG con las mismas variables.

- Integrar imágenes como mapas escaneados en un SIG utilizando coordenadas geográficas.

- Análisis de datos espaciales.

Mientras que del segundo al octavo punto se relatan características propias del uso de he- rramientas SIG, el primero es algo específico de un servicio de biblioteca.

El interés general ha hecho este tipo de información más accesible. Sobre todo en el ámbito de la visualización de datos, ya que nos permiten apreciar datos geográficos de cada vez mayor complejidad. Igualmente, el software sobre información geográfica y los mapas, está cada vez más disponible (Weessies y Dotson, 2013). En relación al tratamiento de la información geográfica, Houser (2006), eleva la noción de servicio SIG a la visualización y análisis de datos espaciales, que conlleva un proceso más complejo que el "diseño de mapas", ya que intervienen relaciones entre diferentes capas y cuestiones geográficas. Ya no se habla, por tanto, de datos bibliográficos, sino de estructuras y datasets.

Parece lógico pensar que, siendo cada vez más frecuente la necesidad de estos datos en docencia e investigación, la gestión de estas colecciones, así como su descripción mediante metadatos, se realice por parte del servicio encargado en la biblioteca universitaria. Para dar respuesta a estas necesidades, y teniendo en cuenta las implicaciones de los datos geográficos, la biblioteca universitaria no puede responder mediante el tradicional modelo de referencia. Sin embargo, si se apuesta por integrar estas colecciones en actividades enmarcadas en la llamada data curation, con fines no solo organizativos sino de preservación y, en último término, de reutilización de los mismos.

En este sentido los datos geográficos responden a una tipología cuya preservación puede, en algunos casos, deberse a la obligatoriedad exigida por las agencias de financiación de proyectos de establecer planes de depósito de datos para su futura reutilización por otros investigadores. Sin embargo, la preservación de este tipo de datos puede suponer un problema en el futuro. The Digital Preservation Coalition en su monográfico sobre preservación de información geográfica (McGarva, Morris y Janée, 2009) fija la problemática de estos datos en su complejidad y variedad de formatos y estructuras, la abundancia de contenido en formatos propietarios, la necesidad de mantener el contexto tecnológico y social en el que el dato se ha creado, la importancia creciente de servicios en la web y de datos dinámicos y efímeros.

La norma ISO 19115-1:2014 regula los metadatos para la descripción geográfica. Sin embargo, como recogen Ariza et al. (2012), éstos no están pensados para facilitar la preservación de esta información. En este sentido, todavía hay escasas iniciativas, entre las que destacan los Archi- 
vos Federales Suizos (Swiss Federal Archives) y la Oficina Federal de Topografía (Swisstopo) que han realizado una propuesta preliminar conjunta para el archivo de datos geográficos (Bos et al, 2010).

En nuestra opinión, para las bibliotecas universitarias, la dificultad reside tanto en el tratamiento integral de esta información, debido a la obsolescencia tecnológica de programas y variedad de estructuras y formatos, como en la elección y planificación del tipo de servicio. Estos pueden desarrollarse, bien en solitario, o integrados en otros afines como los servicios de datos de investigación, con los que pueden compartir espacio, personal y software necesario. Asimismo, la evolución del tradicional servicio de referencia hacia los liaison o embedded librarian, se presenta como un modelo flexible en su puesta en marcha. En relación con este perfil profesional, para la creación de estos servicios no es necesaria solo la dotación tecnológica, sino la cooperación nuevamente entre investigadores y los nuevos geobibliotecarios (Granell, Aguilar y 2013). Estos últimos, habiendo recibido la formación pertinente, darían sentido a la colección de datos geográficos dentro de la organización.

Esta formación pasa por unos niveles de especialización todavía no extendidos en el panorama educativo español. En Estados Unidos, aunque la dotación curricular no es homogénea en el ámbito SIG (el directorio de máster de ALA no lo considera como un criterio de especialización para la búsqueda, por ejemplo); sí se han formulado documentos núcleo sobre los que pivotan las competencias en materia de tratamiento de información geográfica. Es el caso del elaborado por la mesa redonda de la ALA (Map \& Geospatial Information Round Table), titulado Map, GIS and Cataloging / Metadata Librarian Core Competencies (2008). Este documento, también denominado Informe MAGERT, recoge tres tipos de perfil profesional: bibliotecario de mapas, bibliotecario SIG y bibliotecario de catalogación y creación de metadatos. Aguilar y Granell (2015) establecen competencias que se alinean con la figura tradicional del bibliotecario; y que pueden adaptarse a la gestión de la información bibliográfica como referencia, alfabetización, gestión de metadatos, mantenimiento de la colección o visualización de datos.

A pesar de estas iniciativas, creemos que el análisis de la relación y colaboración bibliotecaria en los Servicios de Información Geográfica constituye todavía un reto. Atendiendo a todo esto, el objetivo de este trabajo es doble: en primer lugar identificar los requisitos y competencias en materia de gestión de información geográfica en ofertas laborales del ámbito de
Biblioteconomía y Documentación con el interés de conocer el grado de confluencia con los actuales planes de estudio; y, posteriormente, analizar, los servicios prestados por los SIG asentados en bibliotecas universitarias. El análisis conjunto de ambos resultados, ofrecerá una foto fija de interés que ayude a determinar las características actuales de este tipo de servicios. Son resultados que se retroalimentan, aportando una doble visión, la de las competencias que demandan los empleadores, y las actividades y servicios que prestan estas unidades de información.

\section{Metodología}

Ambos estudios se han llevado a cabo mediante un análisis de contenido de las webs, a través de una serie de indicadores y, posteriormente, de un análisis de datos. Para ambas mediciones se realizaron fichas de análisis de datos independientes. El tratamiento y recogida de los datos se realizó mediante la aplicación Microsoft Excel.

Este análisis emplea como fuentes de investigación preexistentes los trabajos de Kelly (2013) -quien también escoge las universidades mejores posiciones del Ranking de Shanghai para establecer su análisis- y Granell y Aguilar (2013), que analiza ofertas de empleo en el sector.

Para este estudio, en el primer caso se ha partido de la base de datos colaborativa http://jobs. code4lib.org. Se escogió esta fuente, debido al marcado carácter tecnológico de los puestos de trabajo ofrecidos, y además, porque al nutrirse de varias fuentes ofrece un pull de ofertas representativo en cuanto a la situación de ofertas con inclusión de competencias SIG en el ámbito de la Biblioteconomía y la Documentación. A través de la búsqueda por la etiqueta Geographic Information System, se identificaron 109 ofertas comprendidas entre 2007 y 2014 . Una vez determinada la muestra, y recopilados los datos de las ofertas entre el 20 y 25 de marzo de 2015, se llevó a cabo un análisis por las siguientes variables: carácter específico en SIG de la oferta, tipo de perfil profesional requerido, titulación específica en el área de Biblioteconomía y Documentación, necesidad de una titulación vinculada a la Geografía o Geodesia, necesidad de experiencia profesional en la gestión de información geográfica previa y tipo de competencias o conocimientos demandados.

Para la segunda parte -la evaluación de los servicios SIG en bibliotecas universitarias-, se tomaron como muestra las bibliotecas de las 50 primeras universidades del Ranking de 
Shanghai en su versión 2013, por considerarse entidades universitarias de relevancia cuyas bibliotecas pudiesen albergar este tipo de servicios. Se aplicó nuevamente el análisis del contenido de las webs, bajo unos parámetros definidos previamente, a través de la "técnica de simulación" denominada "observación participante", en el que los servicios se evalúan desde la perspectiva del usuario. La unidad de evaluación fue la página web de la biblioteca en su totalidad, por lo que no se han analizaron páginas específicas de bibliotecas sucursales $u$ otro tipo de centros documentales dependientes. La evaluación se repitió dos veces para minimizar los posibles errores, en octubre de 2014 y en marzo de 2015. Los parámetros evaluados fueron:

- Existencia de un servicio específico SIG en la biblioteca. No se contempló solo información explicativa, sino que debía mostrar indicios en la web de la existencia de un servicio, visible y en el que la biblioteca tenga un papel activo, por ejemplo en la gestión de repositorios de información geográfica. No se han valorado aquellos servicios SIG que no contemplen a la biblioteca.

- Grado de integración de la biblioteca en dicho servicio. Se mide a través de una escala de tres niveles: alta, servicio dependiente de la biblioteca universitaria en términos de su gestión; media, si se aprecia una gestión compartida del servicio, pero se observa un papel activo de la biblioteca universitaria; y baja, en el caso de un servicio totalmente independiente de la biblioteca en su gestión, pero en el que se se aprecia asesoramiento o algún vínculo con la biblioteca universitaria (información, tutoriales...).

- Tipo de asistencia ofrecida por la biblioteca: si incluye información de contacto de bibliotecarios especializados, tutoriales, guías temáticas o personal especializado únicamente en GIS.

\section{Resultados}

Se muestran los resultados de acuerdo a los criterios establecidos. Es preciso indicar que ambos estudios de caso tienen su foco fundamentalmente en el ámbito anglosajón, por lo que los resultados y conclusiones pueden no adecuarse a otros contextos, incluido el español.

\subsection{Resultados del análisis de las ofertas} con conocimientos en SIG

De las 109 ofertas de empleo analizadas a través de la etiqueta Geographic Information Sys- tem en el portal http://jobs.code4lib.org/ se obtiene que solo 36 de ellas presentan un título específico sobre información geográfica en la oferta. En las 73 restantes, aparecen competencias relacionadas con la gestión de la información geográfica, pero el título se engloba en áreas temáticas o perfiles profesionales diversos (Figura 1).
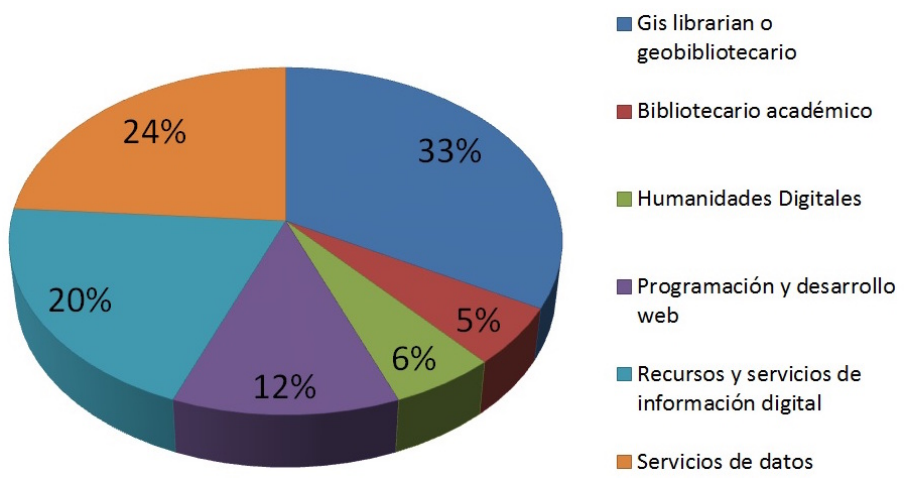

Figura 1. Distribución de las ofertas por ámbito temático de los perfiles demandados

Las 73 ofertas restantes se distribuyen en ámbitos profesionales afines o cuyo tratamiento puede ser conjunto como los servicios de gestión y visualización de datos (24\%). Un $20 \%$ se especifican en ofertas orientadas a los servicios digitales, como la gestión de repositorios, recursos electrónicos y bibliotecas digitales. Asimismo, llama la atención el porcentaje y demanda de perfiles de programador o desarrollador web (formado en lenguajes de programación como Python, por ejemplo), así como otros ámbitos de conocimiento afines como los perfiles de Humanidades digitales que abarcan un $6 \%$ de las ofertas en materia de GIS detectadas. Por último, con un $5 \%$ de representación están aquellas ofertas para las que se demanda un perfil de bibliotecario vinculado con la Educación Superior, en el que una de las competencias sean los SIG.

En relación a las certificaciones y experiencia (Figura 2), es significativo que 70 ofertas, incluyen como requisito una titulación específica en el área de Biblioteconomía y Documentación, especialmente una titulación de posgrado o MLIS (Master of Library and Information Science).Las titulaciones del área de Geografía, Geodesia o Topografía, solo son imprescindibles en 12 ofertas. Sin embargo, sí se requiere en numerosas ofertas un bagaje teórico en la disciplina. Sobre la experiencia profesional específica en un puesto de la gestión de la información geográfica, tan solo 27 ofertas lo demandan. Habitualmente lo que se solicita son destrezas y 
competencias, aunque en ocasiones se escoge la formula combinada de formación académica en Biblioteconomía y Documentación (ByD) y años de experiencia en uso y manejo de SIG.

Experiencia profesional previa GIS

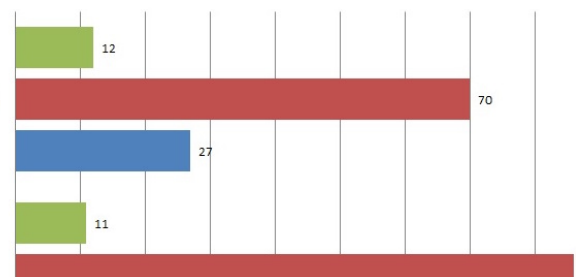

Titulación especifica

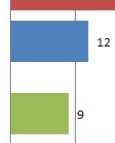

Titulación ByD( LIS)

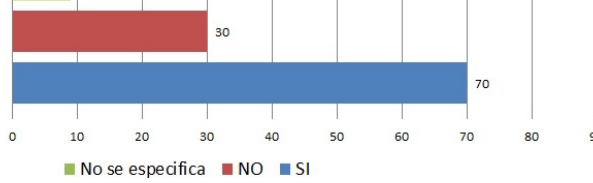

Figura 2. Distribución de las titulaciones y experiencia profesional demandada

En relación a las competencias y conocimientos demandados, estos se han clasificado en cuatro áreas generales (Figura 3).

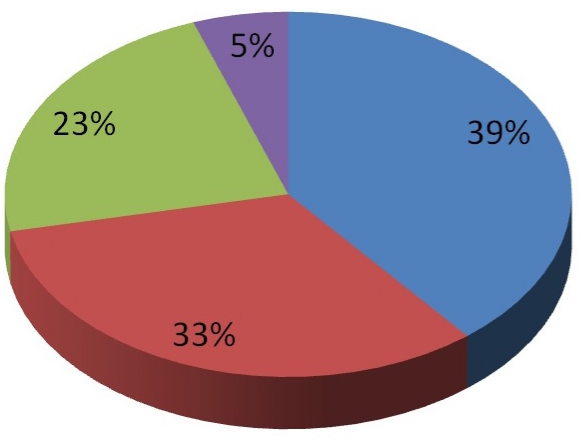

Software y aplicaciones - Conocimientos teóricos - Gestión de colecciones Enseñanza aprendizaje

Figura 3. Distribución de los conocimientos y competencias requeridos en las ofertas de empleo

Un $39 \%$ de las ofertas demandan conocimientos y experiencia en el uso de software específico como ESRI ArcGIS.
Casi al mismo nivel, con un 33\% se demanda conocimientos teóricos en relación a la Geografía y los recursos cartográficos. Este conocimiento, en ocasiones requiere de validación, por lo que parece interesante ofrecer vías intermedias y conjuntas en la formación de los geobibliotecarios. La gestión de colecciones y datos cartográficos, así como su descripción y preservación se demanda en un $23 \%$ de las ofertas señaladas.

Por último, es importante destacar que 104 de las ofertas analizadas de las ofertas provienen del sector universitario, por lo que es frecuente encontrar solicitudes específicas en relación a la integración del bibliotecario en equipos de profesores e investigadores, tareas de alfabetización como la elaboración de material didáctico o guías de recursos en línea (por ejemplo Libguides) o el tradicional servicio de referencia. Estas actividades relacionadas con la enseñanzaaprendizaje se han demandado de manera explícita en un $5 \%$ de las ofertas analizadas.

Dado el predominio de las universidades como demandantes de empleo, es preciso conocer el estado de sus Servicios de Información Geográfica, objeto de estudio del segundo análisis.

\subsection{Resultados del análisis}

de los servicios SIG en las 50 primeras

bibliotecas de las del Ranking de Shanghai

De las 50 bibliotecas analizadas, se ha determinado que 23 tienen un servicio SIG con las especificidades que se requerían al inicio del estudio (Tabla I). De estos servicios, en 13 se detecta una participación alta por parte de la biblioteca, en 4 media, y en 6 considerada como baja.

En el caso del Servicio de Información Geográfica se observa en ocasiones cómo las bibliotecas sí contienen este tipo de materiales, pero estos se encuentras dispersos entre varios servicios o bibliotecas de facultades, lo que ha dificultado una evaluación holística.

\begin{tabular}{lll}
\hline Universidad & Nombre del servicio & Dirección URL \\
\hline Harvard University & Harvard Geospatial Library & http://calvert.hul.harvard.edu:8080/opengeoportal/ \\
\hline Stanford University & Stanford Geospatial Center & https://lib.stanford.edu/gis \\
\hline $\begin{array}{l}\text { University of California, } \\
\text { Berkeley }\end{array}$ & Earth Sciences \& Map Library & http://www.lib.berkeley.edu/EART/gis \\
\hline $\begin{array}{l}\text { Massachusetts Institute of } \\
\text { Technology (MIT) }\end{array}$ & $\begin{array}{l}\text { Geographic Information } \\
\text { Systems (GIS) }\end{array}$ & http://libguides.mit.edu/c.php?g=176295\&p=1160692 \\
\hline Princeton University & Map and Geospatial Information & http://library.princeton.edu/collections/pumagic \\
\hline
\end{tabular}




\begin{tabular}{|c|c|c|}
\hline & Center & \\
\hline Columbia University & Digital Social Sciences Center & http://geodata.cul.columbia.edu/ \\
\hline Yale University & $\begin{array}{l}\text { Geographic Information } \\
\text { Systems (GIS) }\end{array}$ & http://www.library.yale.edu/MapColl/gis_index.html \\
\hline Cornell University & $\begin{array}{l}\text { Cornell University Geospatial } \\
\text { Information Repository }\end{array}$ & http://cugir.mannlib.cornell.edu/about.jsp \\
\hline $\begin{array}{l}\text { University of California, } \\
\text { San Diego }\end{array}$ & Data \& GIS Lab & $\begin{array}{l}\text { http://libraries.ucsd.edu/resources/data-gov-info-gis/data-and-gis- } \\
\text { lab/index.html }\end{array}$ \\
\hline University of Washington & $\begin{array}{l}\text { Geographic Information } \\
\text { Systems (GIS) Lab }\end{array}$ & http://wagda.lib.washington.edu/gislab/ \\
\hline $\begin{array}{l}\text { The Johns Hopkins } \\
\text { University }\end{array}$ & GIS \& Data Services & http://guides.library.jhu.edu/gis \\
\hline $\begin{array}{l}\text { University of Wisconsin - } \\
\text { Madison }\end{array}$ & Gis Services & http://www.geography.wisc.edu/maplib/giservices.php \\
\hline $\begin{array}{l}\text { University of Michigan - } \\
\text { Ann Arbor }\end{array}$ & GIS & http://www.lib.umich.edu/clark-library/gis-at-umich \\
\hline New York University & Data Services & http://nyu.v1.libguides.com/friendly.php?s=GIS \\
\hline University of Toronto & Map \& GIS Services & http://mdl.library.utoronto.ca/collections/geospatial-data \\
\hline Northwestern University & Maps \& GIS & $\begin{array}{l}\text { http://www.library.northwestern.edu/libraries-collections/evanston- } \\
\text { campus/government-information/maps-gis }\end{array}$ \\
\hline Duke University & Data and Visualization Services & http://library.duke.edu/data/gis \\
\hline $\begin{array}{l}\text { University of Maryland, } \\
\text { College Park }\end{array}$ & $\begin{array}{l}\text { U.S. Government Information, } \\
\text { Maps \& GIS Services }\end{array}$ & http://www.lib.umd.edu/gov-info-gis/maps-and-gis/gis \\
\hline $\begin{array}{l}\text { University of British } \\
\text { Columbia }\end{array}$ & GIS Services & http://koerner.library.ubc.ca/services/gis-services/ \\
\hline $\begin{array}{l}\text { University of North } \\
\text { Carolina at Chapel Hill }\end{array}$ & Gis \& Data Services & http://library.unc.edu/services/data/ \\
\hline $\begin{array}{l}\text { University of California, } \\
\text { Davis }\end{array}$ & Map \& GIS Data Collection & https://www.lib.ucdavis.edu/dept/mapcollection/ \\
\hline $\begin{array}{l}\text { University of Southern } \\
\text { California }\end{array}$ & Mapping/GIS Data & $\begin{array}{l}\text { http://guides.lib.ucdavis.edu/content.php?pid=487832\&sid=40005 } \\
\text { 56\#14860012 }\end{array}$ \\
\hline Vanderbilt University & $\begin{array}{l}\text { Scholarly Communications ( } \\
\text { GIS) }\end{array}$ & http://www.library.vanderbilt.edu/scholarly/gis.php \\
\hline
\end{tabular}

Tabla I. Denominación y dirección URL de los 23 servicios de Información geográfica detectados

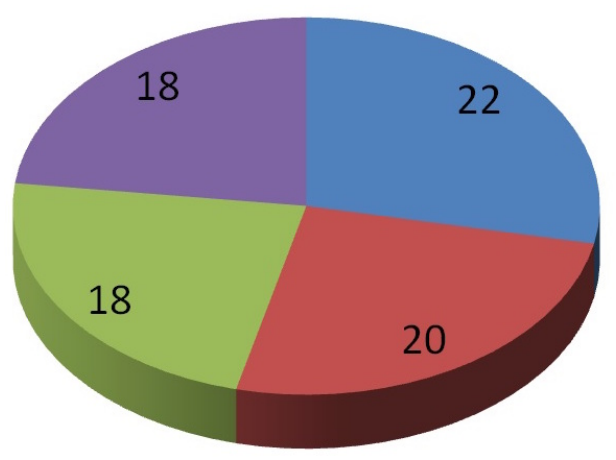

- Información básica de contacto

- Tutoriales

m Guías temáticas específicas

Personal especializado integrado

Figura 4. Tipo de material o personal de asistencia ofrecido por el Servicio de Información geográfica
De estas 23 bibliotecas, se ha detectado una presencia similar en la provisión de servicios de apoyo y ayuda al usuario (Figura 4). La información de contacto específica del servicio SIG está presente en 22 bibliotecas. Le siguen de cerca recursos como tutoriales (sobre todo de software y su aplicación). En materia de personal, llama la atención positivamente como existe un bibliotecario especializado en SIG (o en servicios afines que lo incluyen) en 18 bibliotecas. En el mismo número de centros se elaboran guías temáticas de recursos SIG como ayuda al usuario.

Se da la paradoja, en muchas ocasiones, de que a pesar de que existan servicios y laboratorios de Información Geográfica en las universidades, éstos no contemplan ni enlazan a la biblioteca; sin embargo, esta sí que establece guías de recursos a los mismos, incluyendo 
bibliotecarios integrados para estos temas. Se observa que, en ocasiones, no se dota de entidad al Servicio de Información Geográfica dentro de la biblioteca.

Otra tendencia detectada, es asimilarlo a un Servicio de Información Cartográfica convencional. Muchas páginas, solo enlazan con la colección cartográfica y aportan algunas nociones sobre el software más utilizado, pero un servicio SIG es algo más: ha de requerir tanto en su concepción física como digital de personal especializado y mecanismos de asistencia específicos.

Una visión operativa es integrar estos servicios junto con otros de cariz similar, como los de datos de investigación ( Figura 5). Este criterio de aproximación a la gestión de datos más global también pudo verse en el análisis de ofertas de empleo ( Figura 1).

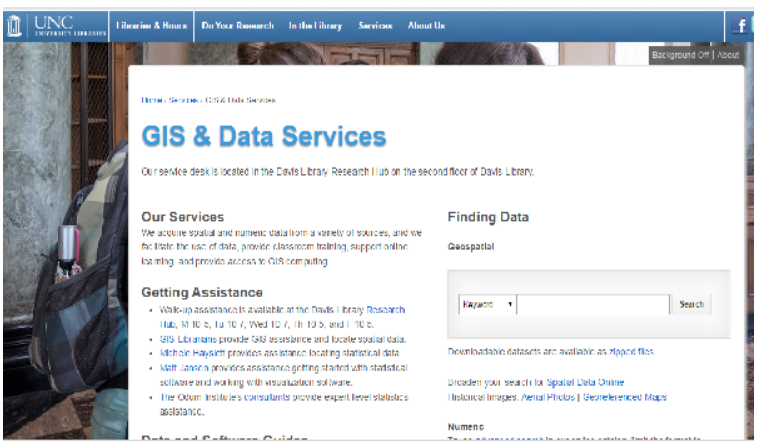

Figura 5. GIS \& Data Services

de la Universidad de North Carolina-Chapell Hill

(Fuente: http://library.unc.edu/services/data/, marzo 2015)

Son ocho las bibliotecas cumplen todos los indicadores establecidos en la evaluación de los servicios SIG: la Universidad de Harvard, Universidad de Stanford, Massachusetts Institute of Technology (MIT), Universidad de California, San Diego, Universidad de Nueva York, Universidad de Toronto, Universidad de Duke, Universidad de British Columbia y Universidad de North Carolina en Chapel Hill.

De la muestra evaluada y a través del análisis personal realizado, se ha detectado que 27 bibliotecas universitarias no contemplan o no muestran específicamente a través de la web este servicio. Al cruzar los datos con las ofertas de empleo analizadas, 18 son las Universidades del Ranking de Shanghai que figuran en las ofertas como empleadores.

La que más ofertas inserta es la Universidad de Stanford, con ocho ofertas laborales relacionadas con servicios SIG entre 2007-2014. Le siguen la Universidad de New York con seis; Cor- nell, Duke el MIT y Yale con tres; Harvard y la Universidad de California San Diego con dos; mientras que del resto se encuentra una oferta por universidad.

Al cotejar los datos de las ofertas de empleo con la evaluación de los servicios de información geográfica, se ha observado la variedad de estructuras que las universidades ofrecen como apoyo al uso de la información geográfica al mismo tiempo, por lo que es frecuente que el puesto de trabajo que se solicita sea de carácter flexible, esto es, no asociado directamente con los servicios SIG in situ, sino que fluctúa desde el tradicional apoyo a docentes e investigadores en departamentos, pasando por la integración en servicios de gestión y preservación de la colección digital hasta perfiles de desarrolladores de software.

Se observa que en muchos casos las ofertas de empleo se orientan a nutrir y catalogar una colección existente, pero en otras se pide personal para la formación y creación de servicios SIG integrales, incluso incorporando programas de Metaliteracy o alfabetización integral para concienciar a investigadores y formadores de las potencialidades de utilizar o incorporar los datos geográficos en sus investigaciones o en sus ámbitos de conocimiento.

\section{Conclusiones}

La relación entre servicios de información geográfica y las bibliotecas universitarias es una realidad que precisa de articulación tanto en modelos de colaboración efectivos como en formación reglada en planes de estudio. Los resultados obtenidos permiten ofrecer una radiografía del estado de los servicios SIG en bibliotecas universitarias en la actualidad, especialmente en el ámbito anglosajón. Ambos estudios ofrecen conclusiones que pueden analizarse de manera entrelazada.

De las ofertas de empleo analizadas en la base de datos colaborativa, se manifiesta una posición favorable hacia la contratación de titulados en Información y Documentación. Un $64 \%$ solicita una titulación específica en nuestro área, generalmente nivel Máster (MLIS en Estados Unidos). Son escasas las ofertas en bibliotecas que soliciten solo titulados en materias como Geografía, Geodesia, Urbanismo o Topografía.

Se detecta la necesidad de una fuerte especialización en materias de tratamiento de datos geográficos y en la propia disciplina a nivel teórico. Esto es un hándicap para titulados en Documentación y un potencial nicho formativo que puede ser cubierto, bien con materias de pos- 
grado, rutas específicas de especialización o titulaciones conjuntas, como por ejemplo la hallada en la Universidad de Wisconsin bajo el nombre MA Geography/MLIS degree, impartido entre la School of Information Studies y el Departamento de Geografía de dicha Universidad.

Las ofertas de empleo analizadas plantean una orientación transversal de los perfiles profesionales. Se observa una perspectiva multidisciplinar en las competencias requeridas, a menudo asociadas con aspectos relacionados como el tratamiento y visualización de datos de investigación o las Humanidades Digitales. Esto se verifica en la segunda parte del estudio, con la aparición de centros y servicios SIG combinados, que ofrecen asistencia en el tratamiento combinado de información diversa.

En cuanto a los servicios SIG en bibliotecas universitarias, resultó difícil su identificación en la web ya que en ocasiones la información se encuentra dispersa entre servicios o bibliotecas sucursales y es difícil detectar la vinculación de la biblioteca universitaria con los mismos. Esta dispersión dificulta una evaluación de carácter holístico como la de este estudio. De las 50 bibliotecas de la muestra, 23 tienen un servicio SIG o disponen de estructuras de apoyo a la información geográfica; de estas, la participación de la biblioteca se consideró alta en 13 de ellas. En cuanto al contenido y servicios, existe una voluntad clara de fomentar a través de las bibliotecas universitarias la alfabetización en SIG gracias a la recopilación de tutoriales específicos sobre software y guías temáticas que recopilan aspectos clave en materia de datos geográficos.

Creemos que es preciso articular un modelo de implantación de servicios de información geográfica efectivo en bibliotecas universitarias, que se incorpore a las nuevas estructuras de creación, tratamiento y preservación datos de investigación existentes. Estos servicios han de trascender el espacio físico, y representar un mode- lo escalable, que sea un eslabón entre el personal bibliotecario y los investigadores. Debido a su flexibilidad, se considera el modelo de Biblioteconomía Integrada o Embedded Librarianship, un prisma adecuado bajo el que ver los la gestión de los sistemas de información geográficos en las bibliotecas universitarias.

\section{Referencias}

Aguilar, E.; Granell, C. (2015). Gestión de datos geográficos en bibliotecas universitarias españolas: Estado de la cuestión. // Revista Española de Documentación Científica,38:2 : e085.

Ariza, F. J; Ariza, R. M; Ureña, M. A; Cortés, J.; Ureña, L. A (2012). Preservación de la Información geográfica: perspectivas y situación en España. // Geofocus. 12, 171-200.

Aufmuth, J. (2006). Centralized vs. distributed systems: academic library models for GIS and remote sensing activities on campus. // Library Trends. 55:2, 340-348.

Bos, M; Gollin, H.; Gerber, U.; Leuthold, J.; Meyer, U. (2010). Preliminary study on the archiving of geodata. Berna. Federal Department of Defence, Civil Protection and Sport, Federal, Office of Topography Swisstopo, Federal Department of Home Affairs, Swiss Federal Archives.

Granell, C.; Aguilar, E. (2013). Se busca geobibliotecario: los datos geográficos entran en la biblioteca. // El profesional de la Información. 22:6, 569-575.

Houser, R. (2006). Building a Library GIS Service from the Ground Up. // Library Trends. 55: 2, 315-326.

Kelly, L. (2013). Maps, Libraries and the "GIS Librarian: an Informal Review of International Cartographic Libraries. // South African Journal of Geomatics. 2:2, 163-174.

Mcgarva, G.; Morris, S.; Janeé, G. (2009). Preserving Geospatial Data. Technology Watch Series report 09-01. New York: Digital Preservation Coalition.

Magert Education Committee (2008). Map, GIS and Cataloging I Metadata Librarian Core Competencies. http://www.ala.org/magirt/publications (2015-03-27).

Weessies, K. W.; Dotson, D. S. (2013). Mapping for the masses: GIS lite and online mapping tools in academic libraries. // Information Technology and Libraries. 32:1, 23-35.

Weimer, K. H.; Reehling, P. (2006). A new model of geographic information librarianship: Description, curriculum and program proposal. // Journal of education for library and information science. 291-30.

Enviado: 2015-03-31. Segunda versión: 2015-07-21. Aceptado: 2015-08-04. 